\title{
Cambios en la eSTRUCTURA DE UN ECOSISTEMA ACUÁtico COMO RESPUESTA A DISTURBIOS ANTRÓPICOS EN LA SELVA ALEDAÑA AL POBLADO DE ATALAIA DEL NORTE, ESTADO DE AMAZONAS, BRASIL
}

\author{
Changes in THE STRUCTURE OF AN AQUATIC \\ ECOSYSTEM IN RESPONSE TO ANTHROPIC \\ DISTURBANCES IN THE RAIN FOREST NEAR TO ATALAIA \\ DO NORTE, AMAZONAS, BRASIL
}

\author{
Guillermo Armando Briceńo Vanegas ${ }^{1}$, Julie Carolina Gallego Herrera ${ }^{2}$ \\ ${ }^{1}$ Universidad de La Salle, Bogotá, Colombia, \\ ${ }^{2}$ Universidad Nacional de Colombia, Bogotá, Colombia
}

Recibido: 23/04/2017 • Aprobado: 08/05/2017

\section{RESUMEN}

Entre 2002 y 2006 se monitoreó el Igarapé de Río Branco, un ecosistema acuático en la Amazonia brasileña en cercanias del poblado de Atalaia do Norte. El propósito fue determinar cómo cambia la estructura del ecosistema en respuesta a disturbios causados por las actividades antrópicas en su entorno. Para evaluar la evolución de las características ecológicas se utilizaron como indicadores: la fluctuación de las variables fisicoquimicas del agua, la oferta y distribución de hábitats acuáticos, las modificaciones estructurales en la fisiografía del cuerpo acuático y la dinámica de la estructura de la comunidad ictica. Las variables fisicoquimicas fueron medidas en campo con equipos YSI33, YSI35 y Aquamerck. La oferta y distribución de hábitats se calculó con una modificación de la metodología de Gorman y Karr (1978). Los cambios fisiográficos del cuerpo acuático se graficaron con el programa Surfer y la estructura de la comunidad ictica se describió a través de la composición, la abundancia relativa y la utilización espacio-temporal de hábitats. Los patrones de distribución espacial y de utilización de recursos tróficos se analizaron por medio de los programas Past y Biodiversity. Los resultados mostraron que, en el transcurso de 4 años, el Igarapé de Río Branco fue afectado por el decremento en la cobertura vegetal de sus bosques aledaños. La oferta y distribución de hábitats se redujo y la fisiografía del cauce mostró una tendencia a la pérdida de heterogeneidad espacial, lo que presionó a las especies icticas a realizar cambios adaptativos que modificaron la estructura ecológica de la comunidad.

Palabras clave: comunidad ictica, disturbios antrópicos, heterogeneidad espacial, igarapé.

${ }^{1}$ gbricenov@unisalle.edu.co, orcid.org/0000-0002-8784-0867

${ }^{2}$ jcgallegoh@unal.edu.co, orcid.org/0000-0002-3836-1800 


\section{Abstract}

Between 2002 and 2006 the Igarapé of Rio Branco, an aquatic ecosystem in the Brazilian Amazon in the vicinity of the town of Atalaia do Norte, was monitored. The purpose was to determine how the ecosystem structure changes in response to disturbances caused by human activities in their environment. Fluctuation of the physicochemical variables of water, supply and distribution of aquatic habitats, structural modifications in the physiography of the body and dynamics of the structure of the fish community, were used as indicators to evaluate the evolution of ecological characteristics. The physicochemical variables were measured in the field with YSI33, YSI35 and Aquamerck equipment. The availability and distribution of habitats were calculated by means of a modification of a method proposed by Gorman and Karr (1978). The physiographic changes of the aquatic body were plotted with the surfer program and the structure of the fish community was described through the composition, the relative abundance and the spatial-temporal utilization of habitats. The patterns of spatial distribution and utilization of trophic resources were analyzed through the Past and Biodiversity programs. In summary, results showed that during 4 years the Igarape of Rio Branco was affected by decrease of vegetal cover of surrounding forests. The availability and distribution of aquatic habitats was reduced and physiography of aquatic body showed a trend towards diminish its spatial heterogeneity and thereby species were forced to make adaptive changes that modified fish community structure.

Key words: anthropic disturbances, fish community, igarapé, spatial heterogeneity.

\section{INTRODUCCIÓN}

La cuenca del río Amazonas abarca más de 6.000 kilómetros cuadrados de bosque pluvial, distribuido entre Brasil, Surinam, Guyana, Venezuela, Colombia, Ecuador, Perú y Bolivia. Es la mayor extensión de selva tropical, considerada como uno de los ecosistemas más maduros y complejos de la biosfera y la mayor reserva de diversidad biológica y étnica de la tierra.

La complejidad de la selva amazónica radica en que, aunque suele interpretarse erróneamente como una única clase de bosque tropical con una cobertura vegetal homogénea, en realidad corresponde a varios tipos de ecosistemas entremezclados e interdependientes dentro de un fino entramado que es necesario considerar, con suficiente detalle, para una correcta aproximación a su conocimiento. Así pues, dentro de la cuenca amazónica se pueden encontrar diversos tipos de ecosistemas particulares como la várzea, los igapó, los igarapé, y la selva de terrafirme entre otros.

La mayoría de ríos amazónicos tienen niveles de fluctuación de hasta una docena de metros en su ciclo anual, de tal manera que las márgenes ribereñas corresponden a extensas planicies inundables, buena parte de las cuales suele quedar sumergida durante los picos de aguas altas. A estos planos inundables se les denomina várzeas y las coberturas vegetales que los ocupan corresponden a un tipo de selva de rápido crecimiento como estrategia de adaptación rítmica a los cambios hidrológicos. Los árboles de la selva de várzea, suelen caracterizarse por tener troncos estirados y angostos que les dan la denominación de varillales, en contraste con la fisonomía de especies arbóreas robustas de grandes troncos que alcanzan hasta 60 metros de altura en la selva alejada de los ríos, comúnmente llamada selva de terrafirme.

En cuanto a ecosistemas acuáticos, al interior de la selva existen lagunas o lagos formados por antiguos meandros fluviales que han quedado aislados con el tiempo, aunque mantienen canales comunicantes con los ríos principales y albergan un tipo particular de selva denominada igapó, que permanece inundada buena parte del año. Por entre el igapó fluyen riachuelos o "caños" de menor caudal que se conocen como igarapés, 
usualmente abastecidos por pequeños afluentes con $\mathrm{flu}$ jo interrumpido o temporal, que afloran en la selva de terrafirme y que reciben el nombre de cachoeiras.

A pesar de ser objeto de interés para la conservación, por sus incalculables riquezas y la generosa oferta de recursos de toda índole, desde el abastecimiento de fuentes hídricas hasta el subsidio de materias primas y de reservas minero energéticas, los ecosistemas amazónicos han experimentado el impacto causado por diferentes actividades, ya sea de subsistencia, como la agricultura, o informales como la extracción maderable y minera que afectan su frágil equilibrio ecológico. En el Estado brasileño de Amazonas, sobre la margen derecha del río Javarí, línea fronteriza con Perú, se encuentra el puerto de Atalaia do Norte, a unos 1.100 kilómetros al suroeste de Manaus y a 70 kilómetros del complejo LeticiaTabatinga en la frontera con Colombia. El poblado, que tiene 80 ańos de creado como municipio en la región noroeste del Estado Amazonas, se ha erigido en las últimas dos décadas como un polo de desarrollo económico en medio de la selva, pero también ha causado impactos confusos sobre el estilo de vida de las tribus autóctonas y degradación en los ecosistemas circundantes.
Esta investigación se realizó en el área de influencia directa de la carretera Pedro Teixeira que une los poblados de Atalaia do Norte y Benjamín Constant y tuvo como propósito determinar los cambios generados en la estructura ecológica del igarapé de Río Branco entre 2002 y 2006 frente a disturbios de origen antrópico como la sedimentación y el lavado de suelos producida por la deforestación, la agricultura y la expansión del casco urbano. Los indicadores utilizados para observar la evolución de este ecosistema acuático durante el periodo de muestreo fueron: el comportamiento de las variables fisicoquímicas, la oferta de hábitats, la configuración fisiográfica del cauce y la estructura espacio temporal de sus comunidades ícticas.

\section{2. ÁREA DE ESTUDIO}

El área de estudio se encuentra próxima al municipio de Atalaia do Norte sobre las márgenes del río Javarí, que conforma la frontera entre Brasil y río Javarí, que conforma la frontera entre Brasil y Perú, al norte del Estado de Amazonas, Brasil. Figura 1.

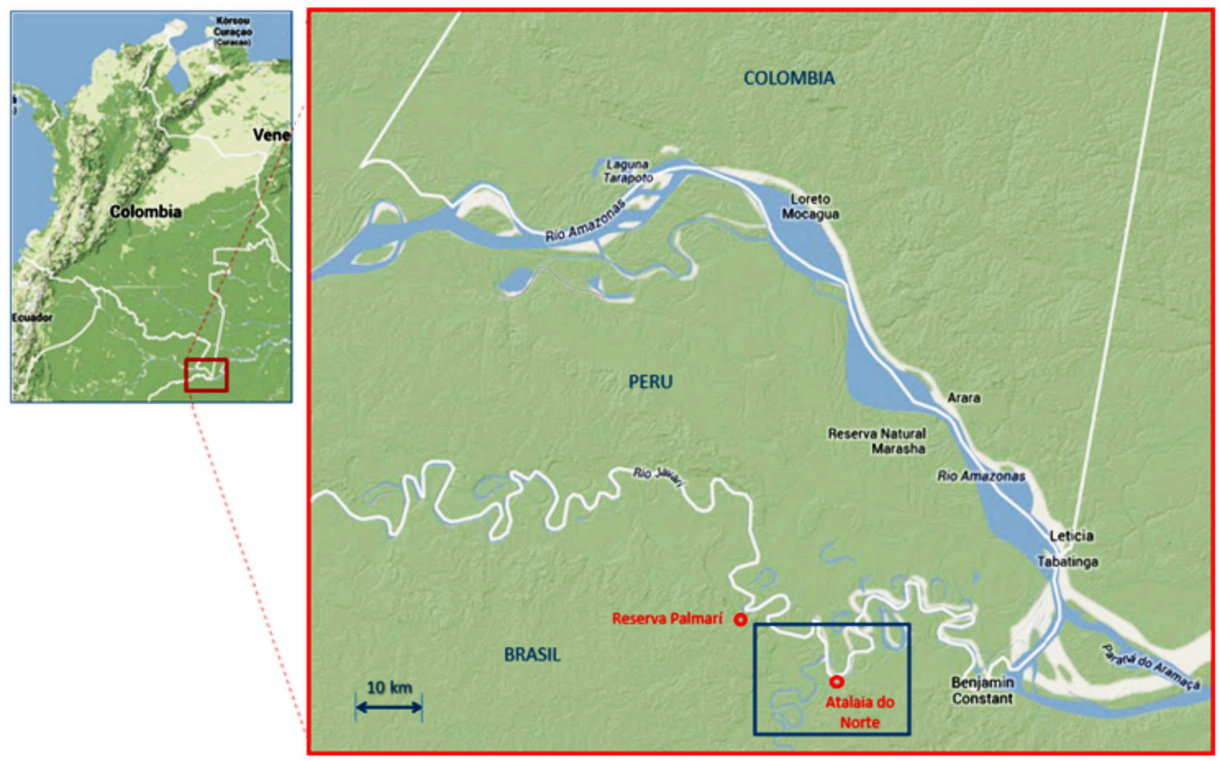

Fig. 1. Área de estudio. Zona fronteriza entre Brasil y Perú siguiendo el curso del río Javarí. En el recuadro se muestra el área de influencia de la carretera Pedro Teixeira en cercanías al poblado de Atalaia do Norte, Estado de Amazonas, en donde se llevó a cabo el muestreo del igarapé de Río Branco.

Fuente: los autores. 
Cambios en la estructura de un ecosistema acuático como respuesta a disturbios antrópicos en la selva aledańa al poblado de Atalaia do norte, Amazonas, Brasil

El clima se caracteriza por valores de precipitación promedio anual de $2.700 \mathrm{~mm}$ y una temperatura de $27^{\circ} \mathrm{C}$. El Javarí es el principal río que atraviesa el área de estudio y es uno de los principales afluentes del Amazonas en la región. Su ciclo hidrológico comprende periodos de aguas altas entre febrero y mayo, un periodo de transición que comienza en junio, hasta llegar a adoptar su mínimo caudal en septiembre y un ascenso de aguas entre octubre y enero.

Las principales unidades de paisaje presentes en el área de estudio se pueden observar en la figura 2.
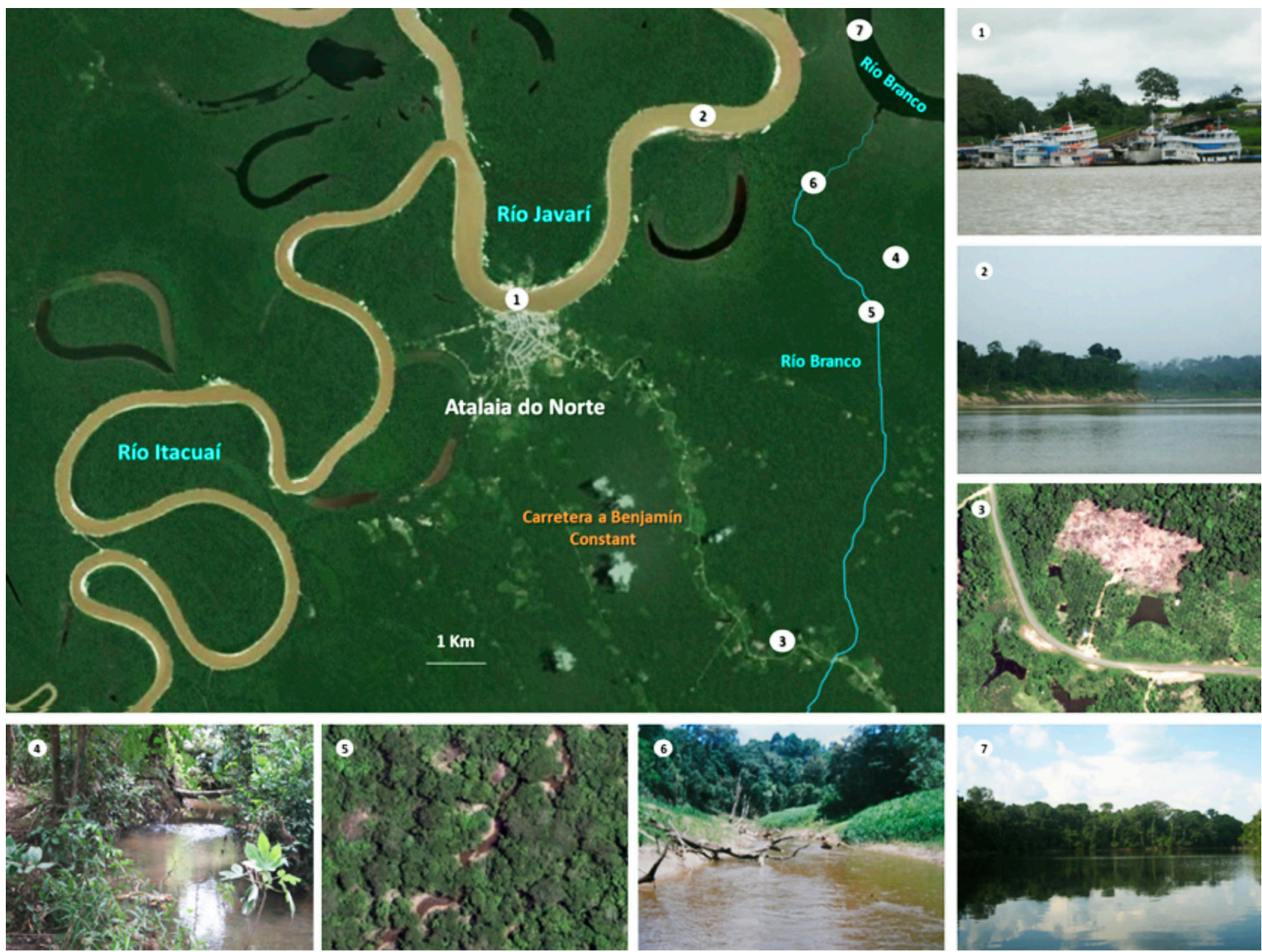

Fig. 2. Unidades de paisaje en el área de estudio para el ańo 2006 (modificado a partir de imagen Landsat). El principal río que atraviesa la región es el Javarí, afluente directo del Amazonas. El Javarí recibe, en cercanías al poblado de Atalaia do Norte, las aguas del río Itacuaí y, además, en la interfase de selva aledaña se forman innumerables igapó e igarapé, dentro de los que destaca el de Río Branco. Fotografías: 1. Puerto del poblado de Atalaia do Norte. 2. Río Javarí. 3. Parches de deforestación en el área circundante a la carretera Pedro Teixeira (foto Landsat, 2006). 4. Cachoeira en inmediaciones a la selva de terrafirme. 5. Vista aérea del curso acuático del igarapé de Río Branco (foto LANDSAT 2006). 6. Canal principal del igarapé de Río Branco. 7. Igapó de Río Branco.

Fuente de las fotografías 1, 2, 4, 6 y 7: los autores. Fotografías 3 y 5: imágenes Landsat. 
Atalaia do Norte es el principal poblado en la región. Para el año 2002 contaba con cerca de 1.1000 habitantes, en su mayoría colonos y foráneos de nacionalidades brasileña, colombiana y peruana. Es también la región en donde se concentra la mayor población de indígenas de las etnias Mayoruna, Corubo y Marubo. Su economía se basa en la extracción maderable y de recursos energéticos y mineros por parte de compañías comerciales y de frentes de colonos que practican la economía informal a partir del comercio de materias primas. Por el incremento de este auge comercial en las dos últimas décadas, los grupos tribales locales, que históricamente habían mantenido un modo de vida basado en sus tradiciones de subsistencia pesquera y agrícola, se han vinculado a la economía extractiva y enfrentan actualmente un problema de transculturización por la pérdida de sus patrimonios ancestrales.

La mayor transformación antropogénica en el área es la carretera Pedro Teixeira que une a Atalaia do Norte con el poblado de Benjamín Constant. Alrededor de este trazado se han derivado ramales de penetración hasta las fuentes de recursos maderables y mineros. La construcción y la posterior penetración de la carretera han implicado la fragmentación de grandes porciones de selva, y los suelos de las áreas deforestadas han sido aprovechados para el establecimiento de cultivos de palma de aceite, y de chagras (brechas practicadas en la selva por indígenas que talan y queman para hacer cultivos transitorios, principalmente de yuca y pińa). La deforestación, a su vez, ha causado el lavado y la erosión de los suelos y estos procesos han generado cambios estructurales en los ecosistemas ribereños del río Javarí, así como en el caso del igarapé de Río Branco.

\section{Materiales y MÉTODOS}

En la zona circundante a Atalaia do Norte, se llevó a cabo un estudio del cauce principal del igarapé de Río Branco, ubicado aproximadamente a $7 \mathrm{~km}$ al este de la población, dentro del área de influencia de la carretera Pedro Teixeira, en las coordenadas $4^{\circ} 24^{\prime} 50^{\prime \prime}$ de latitud sur y $70^{\circ} 09^{\prime} 21^{\prime \prime}$ de longitud oeste.

En un periodo de 4 ańos, se realizaron dos visitas de muestreo en campo: una en 2002 y otra en 2006. Ambas visitas se llevaron a cabo en el mes de julio por corresponder a la etapa del ciclo hidrológico de transición de aguas altas a aguas bajas del río Javarí. En cada una de las visitas se midieron las variables fisicoquímicas del agua, la oferta de hábitats acuáticos, la variación en la configuración fisiográfica del igarapé y los cambios resultantes en la estructura de las comunidades ícticas a través de los parámetros composición, riqueza, abundancia relativa y diversidad.

\subsection{Caracterización fisicoquímica}

La fluctuación de las variables fisicoquímicas del agua, se caracterizó a través de monitoreo in situ, utilizando un equipo YSI33 para determinar $\mathrm{pH}$, temperatura y conductividad eléctrica y un multiparámetro YSI 55 para oxígeno disuelto. Además, se midieron los niveles de nutrientes principales como nitrógeno en forma oxidada $\left(\mathrm{NO}_{2}\right.$ y $\left.\mathrm{NO}_{3}\right)$ y reducida $\left(\mathrm{NH}_{4}\right)$ y fosfatos, utilizando un laboratorio portátil Aquamerck.

\subsection{Hábitats acuáticos}

En 2002 y 2006 se hicieron levantamientos en campo de la estructura tridimensional del cauce del igarapé de Río Branco y de los hábitats acuáticos presentes por medio de una retícula de puntos trazada sobre una franja de 50 metros de largo por 25 de ancho, según una modificación de la metodología propuesta por Gorman y Karr (1978). 

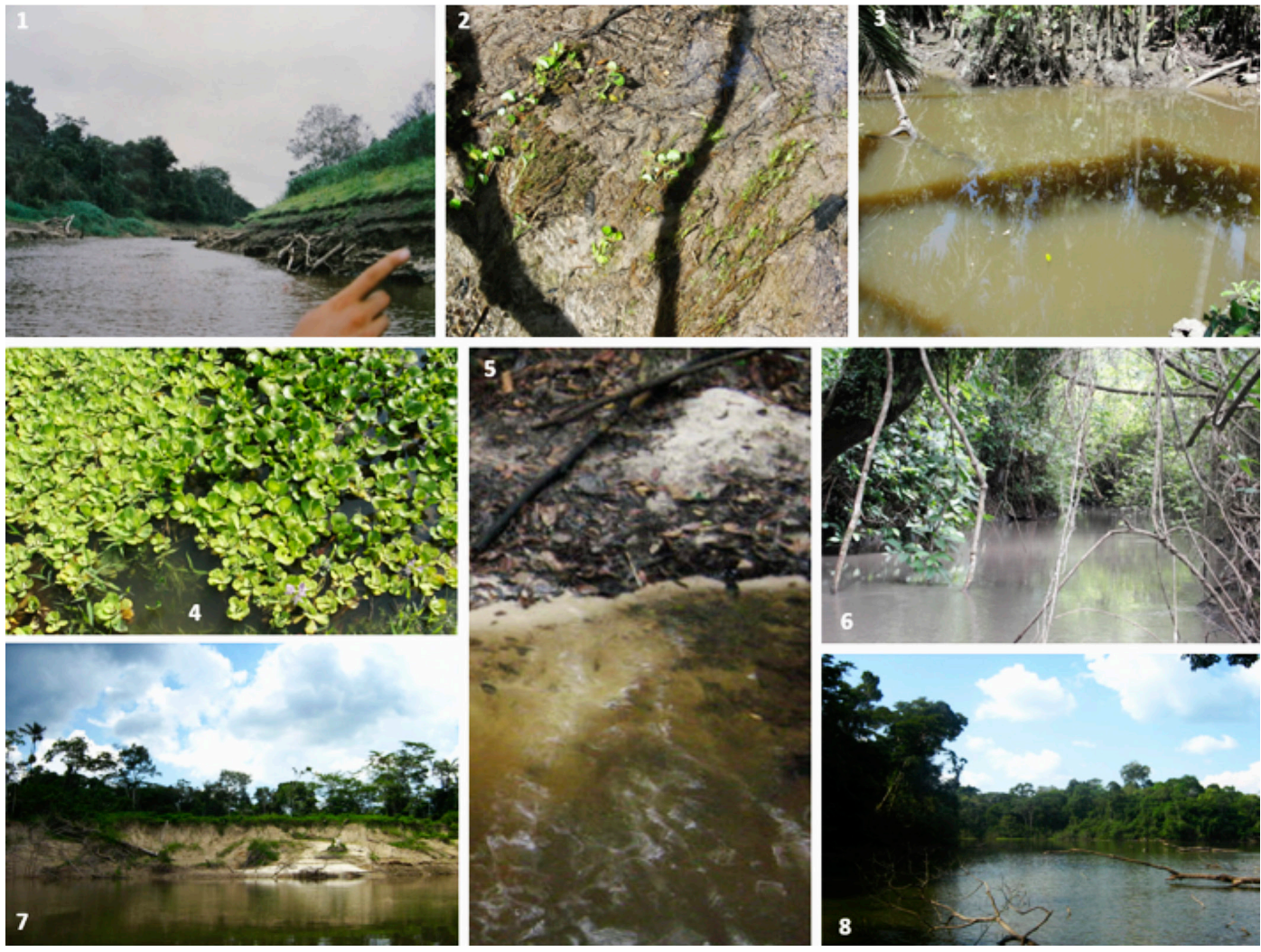

Fig. 3. Tipos de hábitat acuáticos y paisajes asociados al igarapé de Río Branco. 1. Hábitat de canal principal en el periodo de transición a aguas bajas mostrando las fluctuaciones de nivel asociadas al ciclo hidrológico. 2. Hábitat de regadal sobre sustratos arcillosos y pedregosos con macrófitas del género Hydrocotyle asociadas a la corriente. 3. Hábitat de pozo con material vegetal sumergido. 4. Hábitat de vegetación marginal con cobertura de macrófitas flotantes de las especies Pistia stratioides y Eichornia crassipes en las márgenes del igarapé. 5. Hábitat de charcas temporales con abundante materia orgánica y hojas en descomposición sobre sustrato arenoso. 6. Hábitat de plano marginal del igarapé con selva inundable asociada. 7. Vista general del igarapé de Río Branco. 8. Vista general del igapó de Río Branco. Fotografías 1, 5 y 7, redigitalizadas del 2002.

Todas las fotografías son de los autores.

En cada uno de los puntos de la retícula, espaciados un metro entre sí, se midieron la profundidad, la velocidad de la corriente y el tipo de sustrato según granulometría y con ayuda de perfiles batimétricos trazados se determinaron las categorías de clasificación de los hábitats acuáticos como se muestra en la figura 3.

La oferta de hábitat se calculó en porcentaje para cada año de muestreo según las siguientes tipologías: 1. Canal principal; correspondiente a zonas con profundidad homogénea y flujo laminar. 2. Regadales; zonas de escasos centímetros de profundidad, con flujo turbulento sobre sustratos pedregosos o arcillosos que afloran sobre la superficie. 3. Pozos; como hábitats con depresiones o cavidades locales de mayor profundidad y velocidad de corriente reducida. 4. Vegetación sumergida; zonas someras con presencia de macrófitas enraizadas al fondo y gramíneas sumergidas o marginales en forma de gramalotes. 5. Charcas marginales; como estanques temporales separados del canal principal y 6. Áreas marginales de plano inundable.

\subsection{Configuración fisiográfica}

Para cada uno de los años de muestreo se generaron modelos gráficos tridimensionales de la estructura del curso acuático por medio del software Surfer. La comparación gráfica entre años permitió evaluar el impacto sobre el ecosistema usando los criterios de homogeneidad y heterogeneidad estructural. Cómo se ha demostrado en otros estudios (Flecker, 1992; Peres et al., 2010), 
a mayor impacto ambiental sobre un curso acuático hay mayor tendencia del mismo a la homogenización y por ende a la pérdida o merma de la oferta de hábitats y de la diversidad íctica.

Los modelos tridimensionales permiten proyectar tendencias futuras de evolución estructural del cauce del igarapé y establecer de esa manera las estrategias de manejo para su recuperación.

\subsection{Estructura de la comunidad íctica}

En 2002 y 2006 se desarrollaron muestreos de especies ícticas en el igarapé de Río Branco, utilizando artes de pesca como atarraya de $0,1 \mathrm{~cm}$ de ojo de malla y trasmallos artesanales facilitados por nativos mayoruna. Los muestreos fueron dirigidos en periodos de 15 días sobre los 6 tipos diferentes de hábitat tanto en registros diurnos como nocturnos para poder determinar el nivel de ocupación específico. Los ejemplares capturados se mantuvieron en acuarios con suministro de alimento y oxígeno, se fotografiaron y luego de su determinación taxonómica se devolvieron al agua por cuanto no se contaba con permiso de colección biológica. Las determinaciones taxonómicas de las especies ícticas se llevaron hasta donde fue posible al nivel de especie, utilizando las claves taxonómicas de Eigenmann ([1911] 1977; [1922] 1976), Schultz (1944), Sterba (1959), Gery (1964), Lowe-McConell (1987) y Mago-Leccia (1994).
Una vez organizados los datos de capturas, se estableció la composición y la riqueza de la comunidad íctica para cada ańo de muestreo, y se determinaron la aptitud trófica y el nivel de ocupación de hábitat de cada una de las especies más representativas. Para el tratamiento estadístico de los datos, se hizo un análisis de agrupación por similaridad entre especies, con el índice de solapamiento específico de Morisita por medio de los programas Past y Biodiversity.

\section{Resultados}

\subsection{Caracterización fisicoquímica}

En la figura 4 se puede observar la fluctuación de las variables fisicoquímicas tomadas in situ en el igarapé de Río Branco. Todas las variables se registraron en un periodo de 15 días durante el mes de julio de los años 2002 y 2006 respectivamente y se promediaron los resultados de las muestras para presentar un único dato mensual anual.

La temperatura promedio aumentó de 23,2 a 27,5 grados centígrados en el periodo de 4 años. El pH se incrementó desde un valor ligeramente ácido de 5,7 hasta 7,2, mientras que el oxígeno disuelto mostró un decremento de 0,7 miligramos por litro entre las cargas promedio de $2002(6,5 \mathrm{mg} / \mathrm{l})$ y $2006(5,8 \mathrm{mg} / \mathrm{l})$. a

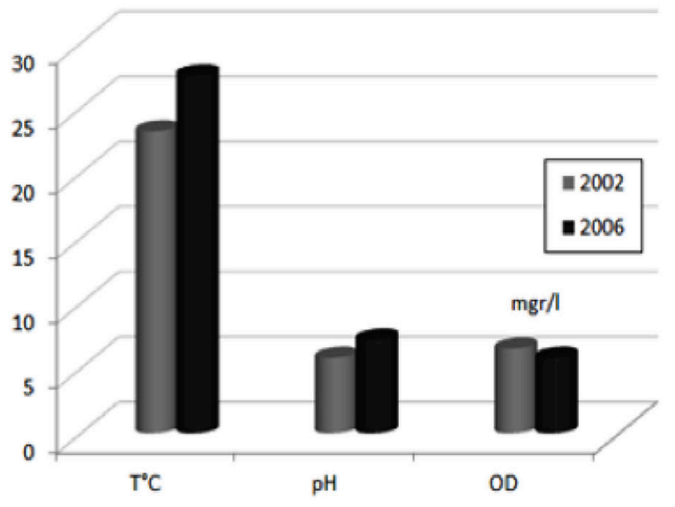

b

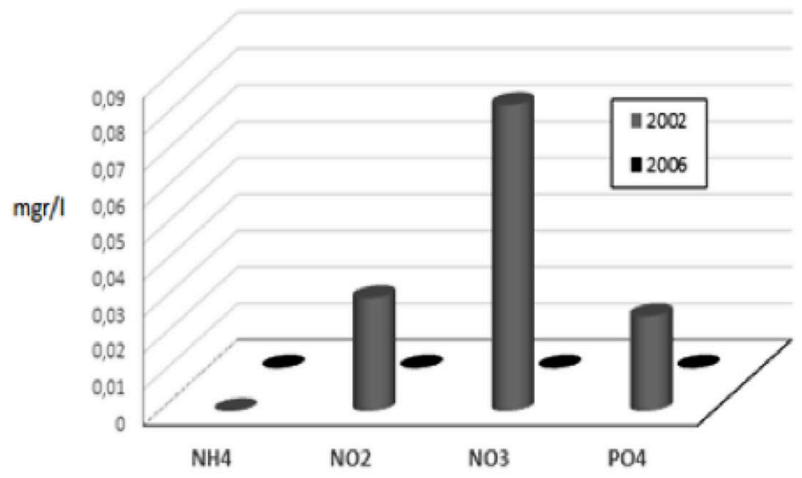

Fig. 4. Comportamiento de las variables fisicoquímicas en el agua del igarapé de Río Branco para los años 2002 y 2006 . En la figura a se muestra la fluctuación de la temperatura en grados centígrados, el $\mathrm{pH}$ y el oxígeno disuelto en miligramos por litro. En la figura b se representa la fluctuación del nitrógeno en forma de amonio, nitritos y nitratos y la del fósforo en forma de fosfatos.

Fuente: los autores. 
Los contenidos de nutrientes variaron de forma diferente. Mientras que en ninguno de los dos muestreos se registraron niveles de amonio, las formas oxidadas de nitrógeno y fósforo que habían resultado negativas en los exámenes de 2002 mostraron incrementos de trazas en $0,031 \mathrm{mgr} / \mathrm{l}$ para nitritos, 0,084 $\mathrm{mgr} / \mathrm{l}$ para nitratos y $0,026 \mathrm{mgr} / \mathrm{l}$ para fosfatos.

En general, las fluctuaciones de las variables químicas revelan la respuesta ambiental a algunos factores de disturbio causados desde el exterior del curso acuático como se discutirá posteriormente. Esta respuesta al disturbio se corroboró por el comportamiento de factores físicos como la conductividad eléctrica que fluctuó entre 169 y $212 \mu \mathrm{S} / \mathrm{cm}$ y los sólidos totales disueltos que se incrementaron de 111,5 a 139,9 .

\subsection{Hábitats acuáticos}

El igarapé de Río Branco mostró un caudal permanente con una anchura fluctuante entre 5 y 12 metros aproximadamente. La profundidad promedio que se registró para el mes de julio, que corresponde al periodo de transición de aguas altas a aguas bajas en ambos ańos, fluctuó entre 2,3 metros en pozos y 0,7 metros en canal principal.

En la franja muestreada del igarapé, se encontraron 5 de las 6 categorías de hábitats para ambos años de muestreo: canal principal, regadales, pozos, vegetación sumergida y charcas marginales. El hábitat de plano inundable sólo se encontró en el año 2002 y cuatro años más tarde no se encontraron vestigios de él.

La oferta de hábitats para ambos años, expresada como el censo porcentual de cada una de las categorías por año, se puede observar en la figura 5.

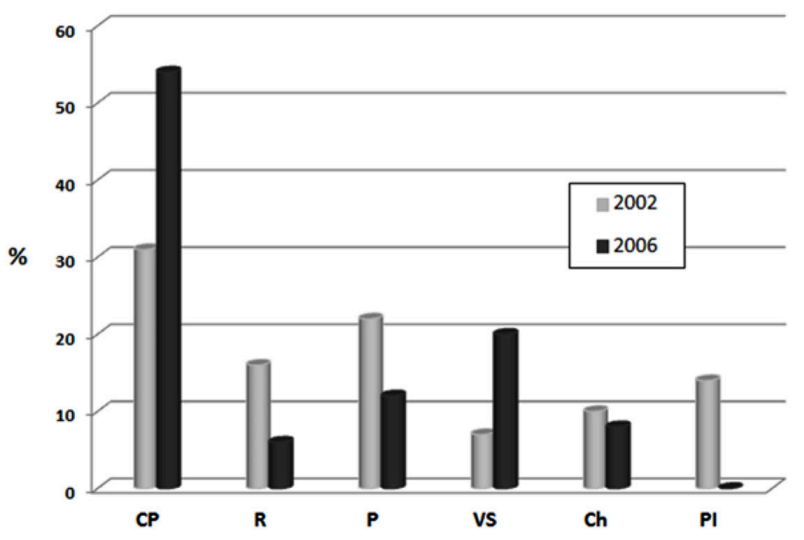

Fig. 5. Oferta de hábitats acuáticos en el igarapé de Río Branco, expresada como porcentaje de presencia de cada una de las categorías para los ańos 2002 y 2006. CP: canal principal, R: regadales, P: pozos, VS: vegetación sumergida, Ch: charcas, PI: plano inundable. Fuente: los autores.

Es evidente que la distribución de hábitats acuáticos varió drásticamente en 4 años. En ambos años de muestreo, el hábitat predominante en el Igarapé fue el de canal principal, pero mientras que para 2002, la distancia entre las frecuencias de todos los tipos de hábitat fue menor, exhibiendo una distribución más equitativa y diversa, para el 2006 el hábitat de canal principal abarcó más del $50 \%$ de la oferta, en detrimento de la frecuencia de los hábitats restantes e incluso desapareciendo la categoría de plano inundable.
Los únicos tipos de hábitat que incrementaron su frecuencia de aparición, durante el periodo de muestreo, fueron los de canal principal (de $31 \%$ a $54 \%$ ) y vegetación sumergida (de $7 \%$ a $20 \%$ ). La oferta del resto de hábitats disminuyó en $10 \%$ para regadales y pozos y $2 \%$ para charcas. El hábitat de plano inundable desapareció para el 2006.

Como se discutirá más adelante, estas tendencias reflejan el impacto de actividades de origen antrópico 
en el área de influencia del igarapé de Río Branco, que afectan su autorregulación ecológica y producen un desequilibrio inequívoco en la estructura de sus comunidades ícticas.

\subsection{Configuración fisiográfica}

En la figura 6 se puede observar la evolución de la configuración fisiográfica del igarapé de Río Branco entre los años 2002 y 2006.

La figura 6a muestra una vista de planta de la configuración batimétrica del igarapé de Río Branco en la que se puede determinar que entre el año 2002 y el año 2006 disminuyeron las cotas de profundidad subacuática y de altura de taludes marginales. En la figura $6 \mathrm{~b}$ las proyecciones en perspectiva del lecho del igarapé demuestran claramente la tendencia del fondo a perder profundidad y a eliminar por sedimentación microhábitats de pozos, barreras y otros accidentes que el sustrato presentaba en el 2002. La figura 6c rebela cómo el espejo de agua también se reconfiguró eliminando compartimientos como islotes y porciones de plano inundable.

En resumen, la comparación anual entre modelos gráficos tridimensionales del igarapé de Río Branco, trazados con el software Surfer, muestra una tendencia a la pérdida de heterogeneidad estructural, lo que concuerda con las tendencias ya revisadas de reducción en la diversidad y oferta de hábitats. Como se detalla en la figura, tanto las cotas de altura de las porciones terrestres marginales al cauce, como las cotas subacuáticas en profundidad, sufrieron modificaciones fisiográficas con un fuerte sesgo hacia la homogenización del suelo ribereńo y del sustrato del fondo, con la consecuente pérdida paulatina de la heterogeneidad espacial.
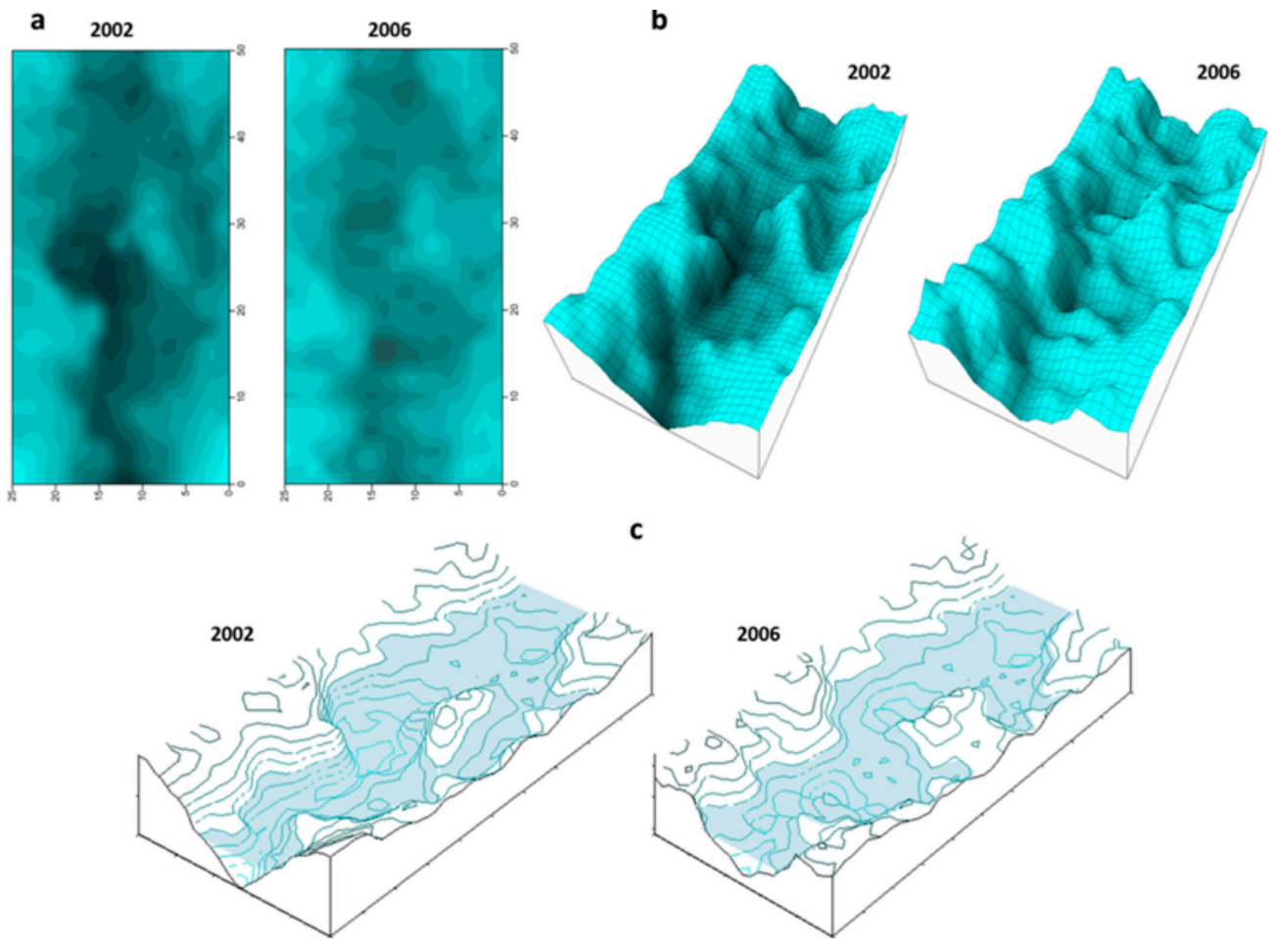

Fig. 6. Configuración fisiográfica del igarapé de Río Branco para los años 2002 y 2006. En la figura a se muestra la vista de planta de la configuración batimétrica. Las zonas oscuras corresponden al ambiente subacuático y las más claras a las zonas ribereñas con taludes marginales sobre el nivel del agua. En la figura b se observa la proyección tridimensional en perspectiva para ambos ańos mostrando la reconfiguración fisiográfica del lecho. La figura c es la representación comparada de la fisiografía del igarapé para ambos años con la cobertura del espejo de agua. Modelos tridimensionales graficados a partir de datos de levantamiento en campo procesados con el programa Surfer.

Fuente: los autores. 


\subsection{Estructura de la comunidad íctica}

En ambos periodos de muestreo se capturaron un total 1.071 ejemplares. En el año 2002, se pescaron 642, representando un $60 \%$ del total del muestreo, mientras que en el 2006 se capturaron 429 correspondientes al $40 \%$ restante. Esta disminución se debió a la reducción de la oferta de hábitats del igarapé por incrementos de sedimentación en los 4 años de muestreo.

La composición de la comunidad íctica estuvo representada en 39 especies pertenecientes a $18 \mathrm{fa}$ milias distribuidas en 5 órdenes. (Anexo 1). De cada uno de los muestreos anuales se seleccionaron las 20 especies más frecuentes de acuerdo con su abundancia relativa, para realizar los análisis correspondientes de ocupación de hábitat y examinar la evolución de la estructura de la comunidad. Estas fueron: Acestrorhynchus falcatus, Astyanax bimaculatus, Charax gibbosus, Creagrutus beni, Hemigrammus marginatus, Curimata spilura, Hoplias malabaricus, Thoracocharax stellatus, Metynnis hypsauchen, Serrasalmus rhombeus, Eigenmannia virescens, Tatia concolor, Corydoras agassizii, Chaetostoma sp, Farlowella gracilis, Hypostomus marginatus, Synbranchus marmoratus, Aequidens tetramerus, Apistogramma eunotus y Cichlasoma bimaculatum.

TABLA 1.

Composición de la comunidad íctica encontrada en el igarapé de Río Branco entre los años 2002 y 2006

\begin{tabular}{|c|c|c|}
\hline Orden & Familia & Especie \\
\hline \multirow{19}{*}{ Characiformes } & Anastomidae & Leporinus Friderici \\
\hline & \multirow{9}{*}{ Characidae } & Acestrorhynchus Falcatus \\
\hline & & Aphyocharax Alburnus \\
\hline & & Astyanax Bimaculatus \\
\hline & & Charax Gibbosus \\
\hline & & Creagrutus Beni \\
\hline & & Hemigrammus Marginatus \\
\hline & & Knodus BETA \\
\hline & & Moenkhausia Oligolepis \\
\hline & & Roeboides Affinis \\
\hline & Crenuchidae & Characidium Fasciatum \\
\hline & \multirow{3}{*}{ Curimatidae } & Prochilodus Nigricans \\
\hline & & Curimata Spilura \\
\hline & & Steindachnerina Bimaculata \\
\hline & Erythrinidae & Hoplias Malabaricus \\
\hline & Gasteropelecidae & Thoracocharax Stellatus \\
\hline & Lebiasinidae & Pyrrhulina Brevis \\
\hline & \multirow{2}{*}{ Serrasalmidae } & Metynnis Hypsauchen \\
\hline & & Serrasalmus Rhombeus \\
\hline \multirow{4}{*}{ Gymnotiformes } & Apteronotiidae & Sternarchorhynchus SP \\
\hline & Sternopygidae & Eigenmannia Virescens \\
\hline & Gymnotidae & Gymnotus Carapo \\
\hline & Apteronotidae & Apteronotus Albifrons \\
\hline \multirow{3}{*}{ Siluriformes } & Auchenipteridae & Tatia Concolor \\
\hline & \multirow{2}{*}{ Callichthydae } & Corydoras Agassizii \\
\hline & & Hoplosternum Thoracatum \\
\hline
\end{tabular}




\begin{tabular}{|c|c|c|}
\hline \multirow{5}{*}{ Siluriformes } & \multirow{4}{*}{ Loricaridae } & Chaetostoma Sp \\
\hline & & Farlowella Gracilis \\
\hline & & Hypostomus Marginatus \\
\hline & & Rineloricaria Lanceolata \\
\hline & Trichomycteridae & Vandellia Sp \\
\hline Synbranchiformes & Synbranchidae & Synbranchus Marmoratus \\
\hline \multirow{7}{*}{ Perciformes } & \multirow{7}{*}{ Cichlidae } & Aequidens Tetramerus \\
\hline & & Apistogramma Eunotus \\
\hline & & Bujurquina $S p$ \\
\hline & & Cichla Ocelaris \\
\hline & & Cichlasoma Bimaculatum \\
\hline & & Crenicichla Sp \\
\hline & & Geophagus Jurupari \\
\hline
\end{tabular}

Fuente: los autores

Las especies Astyanax bimaculatus y Hemigrammus marginatus resultaron ser las más abundantes en ambos años de muestreo, abarcando el 22\% y el $13 \%$ del total de capturas, respectivamente. Como se verá en la sección posterior, este dato cobra mucha importancia en cuanto al análisis de ocupación de hábitat y de utilización de recursos tróficos, si se tiene en cuenta que ninguna de las especies restantes superó el 6\% de abundancia relativa.

En cuanto a la utilización de los recursos alimenticios, las 8 especies a las que se les pudo determinar claramente la aptitud trófica se dividieron en generalistas y especialistas de la siguiente manera: 1. Generalistas. Especies que pueden utilizar más de un ítem alimenticio según la oferta de la estación hidrológica. Dentro de este grupo se clasificaron a los omnívoros comedores de insectos, larvas y semillas como A. bimaculatus. 2 Especialistas. Especies que derivan su sustento de un ítem trófico en particular como es el caso $A$. eonotus, que come hojas y materia orgánica vegetal, C. spilura, que filtra microorganismos del barro, M. hypsauchen, que se nutre de material vegetal sumergido, $H$. marginatus y $C$. bimaculatum, que aprovechan las comunidades de algas microscópicas, o los depredadores como $H$. malabaricus y $S$. rhombeus.

En general, como se puede observar en la figura 7 , el uso del espacio por parte de las especies adaptadas a diferentes hábitats tuvo cambios pronunciados entre los muestreos de 2002 y 2006. 

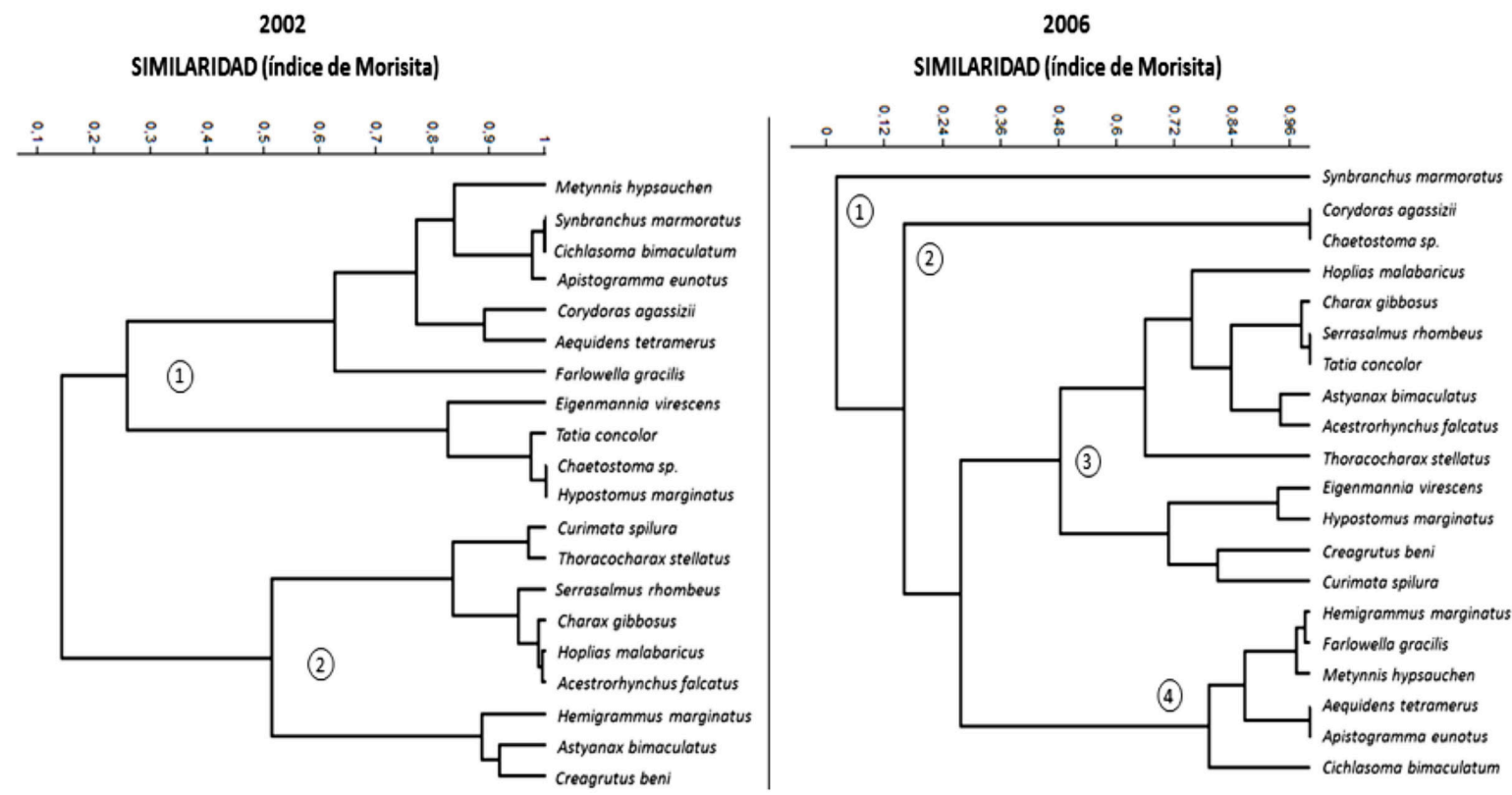

Fig. 7. Análisis de agrupación por similaridad entre especies, según el nivel de ocupación de hábitat para cada uno de los años de muestreo, utilizando el índice de Morisita. Para el año 2002 aparecen dos grupos discriminados por su utilización del hábitat. Estos grupos se reordenan de forma diferente en el 2006 modificando la estructura de la comunidad íctica. Datos procesados con los programas Past y Biodiversity.

Fuente: los autores.

Para el ańo 2002 la estructura de la comunidad íctica revela dos grupos de especies discriminados con una similaridad de 0,15 . ( $85 \%$ de diferencia entre sí). Cada uno de los dos grupos mayores reúne especies empaquetadas a su vez en pequeñas asociaciones de acuerdo con su solapamiento en el espacio del hábitat, el ritmo de actividad o el ítem alimentario. Por ejemplo, dentro del grupo 1 aparecen relacionadas a un $98 \%$ de similaridad, Tatia concolor con Hypostomus marginatus y Chaetostoma sp, todas ellas, especies frecuentes en los hábitats cercanos al lecho del igarapé, ya sea en fondos blandos asociados a profundidad como la primera o de preferencia a regadales como las dos últimas. Otro ejemplo interesante es el de las especies Synbranchus marmoratus, Cichlasoma bimaculatum y Apistogramma eunotus, que se agrupan a un $98 \%$ de similaridad por coincidir en un hábitat que les ofrece el tipo de sustrato que suelen aprovechar como es el de material vegetal sumergido y materia orgánica en descomposición.

El grupo 2 asocia claramente especies afines en un $50 \%$ de similaridad, ya sea, por su abundancia relativa en el hábitat de canal principal (Hemigrammus marginatus con Astyanax bimaculatus y Creagrutus beni a un $87 \%$ ), por sus hábitos alimenticios (Curimata spilura con Thoracocharax stellatus a un $96 \%$ ) o por ocupar el tope de la cadena alimenticia como depredadores normalmente abundantes en el hábitat de pozos (Serrasalmus rhombeus con Charax gibbosus, Hoplias malabaricus y Acestrorhynchus falcatus a un $95 \%$ ).

El muestreo realizado en el año 2006, arroja evidencias de que la distribución espacial (hábitats) o temporal (periodos de actividad) de las especies, así como su abundancia relativa, cambian de tal forma que toda la estructura de la comunidad íctica se reconfigura en función de las modificaciones fisiográficas que el igarapé ha experimentado en el transcurso de los cuatro años. La sedimentación progresiva observada en el lecho durante el periodo de muestreo, causó pérdida de la heterogeneidad espacial del cuerpo acuático y por consiguiente la oferta de hábitats se redujo, obligando a las especies ícticas a adaptarse a espacios y rangos de tiempo diferentes a los que suelen utilizar facultativamente. 
El análisis del solapamiento de hábitats según el índice de Morisita, muestra que para esta época la comunidad se ha reorganizado en dos grandes grupos (grupos 3 y 4), además de una pequeña asociación entre las especies Corydoras agassizii y Chaetostoma sp., y una especie que ha quedado totalmente discriminada de la comunidad que es Synbranchus marmoratus, debido a que en cuatro años cambió de hábitat por completo.

Las especies que en el año 2006 aún permanecen asociadas, con un grado de similaridad del $48 \%$, son las correspondientes a los depredadores de hábitat de pozo y los generalistas que utilizan el hábitat de canal principal (grupo 3), y los usuarios habituales de los hábitat de vegetación sumergida y plano inundable (grupo 4), que debido a los disturbios acaecidos en el igarapé tuvieron que reagruparse todos en el hábitat de vegetación sumergida ante la desaparición inminente del hábitat de plano inundable por la sedimentación progresiva del lecho.

\section{Discusión DE RESUlTAdos}

\subsection{Caracterización fisicoquímica}

El cambio del ambiente fisicoquímico en el igarapé de Río Branco durante los cuatro años de muestreo, representa claramente una respuesta paulatina del cuerpo acuático a disturbios de origen antrópico que han venido impactando el área de influencia que rodea al poblado de Atalaia do Norte y de la carretera Pedro Teixeira, principalmente el aporte de sedimentos al cuerpo acuático.

El aumento del $\mathrm{pH}$ está relacionado con la merma de cobertura vegetal, pues el carácter ligeramente ácido del agua en el 2002, obedecía al continuo aporte de materia orgánica acarreada desde el bosque de galería y su consecuente liberación de taninos ácidos a la corriente al descomponerse. En vista de que, en cuatro ańos, el bosque marginal se ha replegado, la radiación solar ha incrementado la temperatura del agua en muchos tramos, lo cual ha producido una reducción en el nivel de oxígeno disuelto, que explica, además, el registro nulo de especies oxidadas de nutrientes como $\mathrm{NO}_{2}, \mathrm{NO}_{3}$ y $\mathrm{PO}_{4}$ para el año 2006.

Paralelamente a las lecturas de las especies químicas, el aumento de la conductividad y de los sólidos disueltos, induce a pensar que el Igarapé está experimentando un proceso paulatino de sedimentación por lavado y arrastre de suelos aledaños a la corriente ante el desgaste de los taludes laterales y la ausencia de barreras efectivas de vegetación que impidan este proceso erosivo tanto en las riberas como en los hábitats subacuáticos.

\subsection{Hábitats acuáticos}

La desaparición del hábitat de plano inundable en el año 2006, demuestra el efecto erosivo de la configuración fisiográfica del igarapé en sus márgenes laterales. Con la pérdida de este hábitat se reduce también la posibilidad de encontrar charcas temporales, pues la topografía de los taludes se hace restrictiva para generar cubetas o cavidades por fuera del canal principal.

La pérdida de estos ambientes, no solo redujo la oferta de hábitat para la fauna acuática, sino que trajo consigo una afectación permanente, que obligó a la mayoría de especies ícticas a utilizar su capacidad adaptativa para re-ensamblarse dentro de una nueva configuración estructural, sin que el total de ellas lo lograra con éxito. De hecho, las especies generalistas, con una mayor plasticidad conductual, buscaron nuevos hábitats en donde aprovechar los recursos disponibles, mientras que aquellas sometidas a un mayor grado de especialización no volvieron a ser registradas al final de los cuatro ańos de muestreo.

El aumento en la distribución porcentual del hábitat de canal principal se debió entonces a un proceso de homogenización de espacios subacuáticos reforzado por la pérdida de plano inundable y la reducción de charcas, en detrimento de la frecuencia de pozos y regadales. La equitabilidad en la presencia de los 6 tipos de hábitats descritos en el 2002, se transformó en un desequilibrio en la oferta de los mismos, 
que desestabilizó la estructura de la comunidad íctica para el año 2006.

\subsection{Configuración fisiográfica}

La configuración fisiográfica del igarapé, cambió drásticamente en cuatro ańos, como respuesta a los disturbios generados sobre el suelo y la cobertura vegetal del bosque de galería. El principal disturbio observado fue el incremento de las cargas de sedimentación sobre el cuerpo acuático, provenientes de la erosión y la deforestación causadas por la ampliación de la carretera Pedro Teixeira, en conjunto con la expansión del casco urbano de Atalaia do Norte y el despeje y fragmentación de selva para practicar cultivos permanentes de palma de aceite y chagras de agricultura itinerante de yuca y pińa.

La reducción de cotas de altura en las márgenes y de profundidad en los accidentes del lecho, es el resultante de un proceso de desgaste de barreras subacuáticas y de depositación de sedimentos que solo puede responder a la eliminación o merma de coberturas arbóreas y arbustivas. Estas son, en últimas, las que protegen el curso acuático de la erosión y del lavado, debido a que incrementan la retención de suelos ante eventos hidrológicos severos como el aumento de la velocidad de la corriente y del caudal en las épocas de máxima precipitación.

La tendencia presentada en la comparación de los modelos gráficos tridimensionales es a incrementar la homogeneidad espacial del cuerpo acuático, tanto en profundidad como en superficie. Es de esperar que, si la tendencia marcada prevalece, la heterogeneidad espacial natural va a perderse y por consiguiente la diversidad de hábitats y de especies ícticas se reducirá con consecuencias imprevisibles para el equilibrio ecológico del igarapé y de sus ecosistemas adyacentes; más aún, si se tiene en cuenta que este ecosistema particular no solo guarda conexión con los de várzea en el área de influencia, sino que alimenta, desemboca y retroalimenta permanentemente a un ecosistema lacustre como es el igapó.

\subsection{Estructura de la comunidad íctica}

Es muy importante señalar que la mayoría de las especies de la comunida íctica ha respondido de las conclusiones de otras investigaciones desarrolladas en pequeños ríos de la Orinoquía (Briceño, Galvis \& Guillot, 1996; Gery, 1964), y en otras corrientes de bosque tropical (Guilliam, Fraser \& Akins-Koo, 1993; Winemiller, 1990; Aguiar Peixoto, Walker Nelson \& Wittmann, 2009; Angermeier \& Karr, 1994).

En la figura 8, se muestra la evolución de la abundancia relativa de las 8 especies que mejor representaron la dinámica trófica de la comunidad íctica, en relación con el cambio de los patrones espaciales en el igarapé de Río Branco durante el periodo de muestreo.

Si bien A. bimaculatus suele ser una especie prolífica que representó más del 30\% de la abundancia numérica de toda la comunidad, su abundancia relativa se redujo en cuatro ańos prácticamente a la mitad. Cabe aclarar que esta especie suple sus requerimientos alimenticios a partir de insectos, larvas y semillas que son aportados por árboles y arbustos del bosque marginal. Cuando los ríos o cursos acuáticos reciben los insumos energéticos desde los ecosistemas adyacentes y no de su propio cauce, se dice que tienen un metabolismo alóctono en lugar de autóctono. Por lo tanto, la población de A. bimaculatus se vio fuertemente impactada por la merma de la cobertura boscosa que era la fuente de sus recursos alimenticios preferidos. Lo mismo ocurrió con A. eonotus, cuya fuente de alimentación son las hojas sumergidas caídas desde los árboles. 

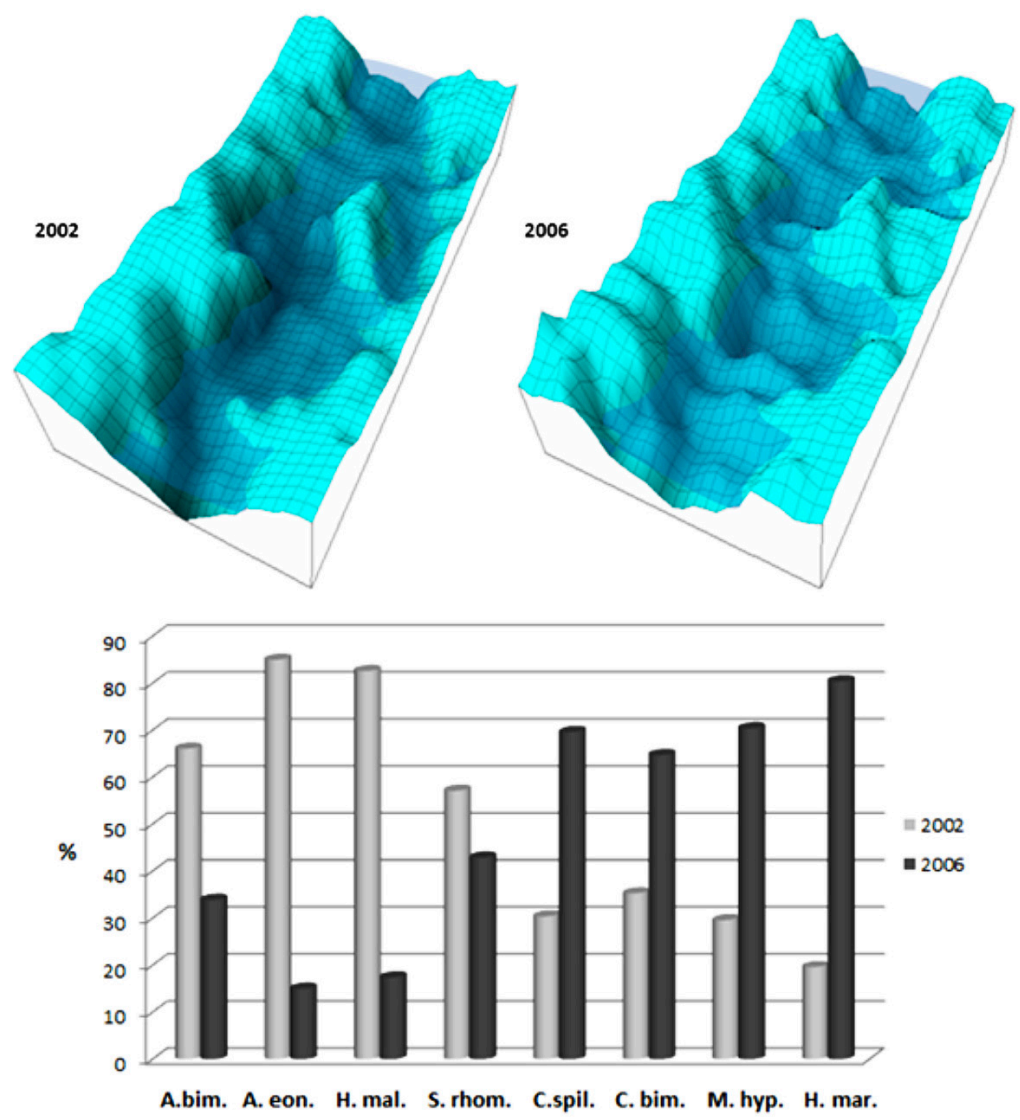

Fig. 8. Evolución de la abundancia relativa de las 8 especies ícticas más representativas de la dinámica trófica de la comunidad, en relación con el cambio de los patrones espaciales en el igarapé de Río Branco entre 2002 y 2006. A. bim: Astyanax bimaculatus, A. eon: Apistogramma eonotus, H. mal: Hoplias malabaricus, S. rhom: Serrasalmus rhombeus, C. spil: Curimata spilura, C. bim: Cichlasoma bimaculatum, M. hyp: Metynnis hypsauchen, H. mar: Hemigrammus marginatus.

Fuente: los autores.

Es muy probable que la disminución de recursos alimenticios de origen alóctono, haya sido la causa de la disminución de la abundancia de $A$. bimaculatus y $A$. eonotus. No obstante, se sabe que todos los ríos y cuerpos acuáticos tropicales funcionan con un metabolismo mixto recibiendo aportes energéticos tanto de su propia estructura acuática como de los ecosistemas aledaños, de tal forma que las especies encuentran en ello un alivio a las tensiones que pueda generar la escasez temporal de algún recurso trófico en particular, lo que explica la prevalencia de estas especies afectadas.

Las otras dos especies que redujeron su abundancia relativa para el 2006 fueron $S$. rhombeus y $H$. malabaricus. Dado el carácter depredador de ambas especies, es muy probable que las poblaciones hayan tenido que dispersarse por todo el espacio efectivo del cuerpo de agua, tanto en extensión como en profundidad, ante la disminución ostensible del hábitat de pozos en donde suelen encontrarse al acecho de sus presas.

En contraste con las anteriores, especies como $C$. spilura, C. bimaculatum, M. hypsauchen y H. marginatus, robustecieron fuertemente sus poblaciones en el igarapé. Para C. spilura, el aumento de la sedimentación probablemente implicó un mejoramiento de su aptitud trófica ya que es una especie comedora de barro del cual filtra microorganismos bentónicos. $M$. hypsauchen suele alimentarse de material vegetal flotante o de macrófitas sumergidas, hábitat que se incrementó en el igarapé por la incidencia directa de la radiación solar sobre el espejo de agua. En el caso de 
C. bimaculatum y $H$. marginatus, ambos comedores de algas microscópicas, el aumento de colonias algales en el agua por la ausencia de sombra del bosque de galería potenció sus condiciones adaptativas, mejoradas aún más en el caso de $H$. marginatus por su reconocido ímpetu reproductivo.

En general, para toda la comunidad íctica, la urgencia de supervivencia en un ambiente tan especializado y competido, supuso un reto adaptativo enorme con estrategias como el traslado de especies de la familia Cichlidae: A. eonotus, $A$. tetramerus y $C$. bimaculatum, al hábitat de macrófitas y vegetación sumergida ante la desaparición de su antiguo hábitat preferido de plano inundable. Aún más severo fue el caso de $S$. marmoratus que para el 2006 solo se encontró en fondos fangosos de la vegetación cuando solía aparecer registrado en taludes del plano inundable cuatro años atrás. Otros trabajos reportan tendencias similares de reorganización espacial de las especies ícticas ante disturbios sostenidos (Briceño, 1994; Angermeier \& Schlosser, 1989).

A pesar de que muchas especies encontraron estrategias para prevalecer en los diferentes ambientes del igarapé, es innegable que la reconfiguración estructural forzada por los disturbios de origen antrópico ya mencionados en este capítulo, causa en este ecosistema acuático un desequilibrio que va a tener consecuencias inciertas en su evolución futura.

\section{Conclusiones}

El igarapé de Río Branco es un ecosistema complejo estructuralmente, rico en biodiversidad íctica y en ambientes propicios para el desarrollo de la biota selvática en general. Sin embargo, con esta investigación quedó en evidencia su alta fragilidad frente a impactos ocasionados por actividades humanas.

La pérdida de cobertura vegetal, causada por la penetración humana en el área de influencia de la carretera Pedro Teixeira, constituyó la mayor fuente de disturbios para el igarapé. Para el año 2006, los usos de suelo que se registraron en áreas deforestadas variaron desde la apertura de chagras, la práctica de cultivos de palma de aceite, y la expansión del casco urbano.

El mayor agente antrópico que afecta la estructura del igarapé de Río Branco, es la sedimentación, a partir del lavado y erosión de suelos por la deforestación y fragmentación del bosque. Esta conclusión se sustenta en el hallazgo de tramos del cauce en donde el bosque de galería se ha mermado de tal forma que los procesos físicos como la remoción de taludes y el lavado por arrastre de los suelos ribereńos, han causado reconfiguración de la fisiografía del curso acuático y una alteración en la oferta y calidad de hábitats que ha afectado la estructura de la comunidad íctica.

Ya en trabajos anteriores se constató que la pérdida paulatina en la cobertura de selva de la várzea del río Javarí, responde a agentes causantes relacionados con la población humana, ya sea por actividades de agricultura itinerante ancestral de los grupos étnicos, o, por el avance de actividades extractivas practicadas por frentes de colonización (Briceño, Briceño \& Gallego, 2012). Estas mismas tendencias se observan a gran escala en toda la Amazonía (Armenteras et al., 2006).

A pesar de los impactos recibidos por el ecosistema acuático, es posible plantear alternativas de conservación que permitan afianzar la recuperación estructural y funcional del igarapé y de los ecosistemas interconectados con él. Sin embargo, es determinante descartar acciones que convoquen simplemente a la protección de los nacimientos o cabeceras de las fuentes hídricas, ya que los igarapé de la Amazonia no se comportan de acuerdo a la teoría clásica del continuum (Vannote et al., 1980), según la cual, los ríos tienen un desarrollo fisiográfico longitudinal que comienza con condiciones heterogéneas en su nacimiento y van adoptando una homogeneidad progresiva a medida que transcurren hasta su desembocadura. Por el contrario, los igarapé de várzea mantienen a lo largo de todo su curso una estructura compleja, cuyos microambientes responden más a un ordenamiento en parches que a un continuo longitudinal. 
De esta manera, por su estructura espacial heterogénea, la biodiversidad asociada al igarapé se sustenta en la estabilidad de relaciones bióticas que dependen adaptativamente de ciclos ambientales rítmicos para garantizar su integridad ecológica. Gracias a ello, en el igarapé puede llegar a encontrarse más diversidad de especies ícticas que en los ríos de mayor porte, pero al mismo tiempo es un ecosistema más sensible a los disturbios repentinos. Por contraste, en los ríos de mayor porte se puede encontrar más biomasa, más abundancia relativa por especie, pero menos diversidad debido a que suelen exhibir menor heterogeneidad espacial que un igarapé.

Para lograr la recuperación del igarapé de Río Branco, y de otros ecosistemas en situaciones similares, se recomienda regular las prácticas de chagras de cultivos transitorios, evitar la expansión de cultivos de palma por parte de las autoridades ambientales y de las gobernanzas indígenas, revegetalizar las márgenes de amortiguación de la carretera y reducir los disturbios antrópicos locales para reactivar los procesos sucesionales del bosque de galería. La resiliencia del ecosistema tolera impactos producidos por disturbios naturales sutiles, con un ritmo cíclico si se permite este proceso sucesional (Wittman, Junk \& Piedade, 2004), pero no puede responder ante disturbios súbitos e imprevistos como los generados por actividades antrópicas (Flecker, 1992).

A una mayor escala, es muy importante trazar planes de conservación de espacios protegidos, que abarquen las redes de drenaje de pequeñas corrientes, pues son las que sostienen el balance hídrico y los pulsos rítmicos de equilibrio ecológico entre las comunidades del bosque pluvial tropical (Thieme, 2007; Peres, 2010).

\section{REFERENCIAS}

Aguiar Peixoto, M., Walker Nelson, J. B. Wittmann, F. (2009). Spatial and temporal dynamics of river channel migration and vegetation in central Amazonian white-water floodplains by remote-sensing techniques. Remote Sensing of environment, 113, 2258-2266.
Angermeier, P.L. Karr, J. R. (1994). Fish communities along environmental gradients in a system of tropical streams. Environmental Biology of Fishes, 9(2), 117-135.

Angermeier, P.L., Schlosser, I. J. (1989). Species-area relationships for stream fishes. Ecology, 70, 1450-1462.

Armenteras, D. Rudas, G., Rodríguez, N., Sua, S., Romero, N. (2006). Patterns and causes of deforestation in the Colombian Amazon. Ecological Indicators, 6, 353-368.15.

Briceño. G. (1994). Distribución espacial de la comunidad íctica como respuesta a la oferta alimenticia en un cańo del piedemonte llanero. Diógenes, 1(2), 151-166.

Briceño, G., Briceño, J. Gallego, C. (2012). Evaluación del deterioro ambiental de hábitats asociados a ecosistemas fluviolacustres de la cuenca del rio amazonas. Epsilon, 13, 203-219.

Briceño, G., Galvis, G., Guillot, G. (1996). Descripción espaciotemporal de la oferta de coriotopos y caracterización fisicoquímica de tres sistemas lóticos del piedemonte llanero. Diógenes, 3(1), 41-62.

Eigenmann, C. H. (1977 [1911]). The freshwater fishes of British Guyana. Amsterdam: Limnaeus press.

Eigenmann, C. H. (1976 [1922]). The fishes of western South America. Amsterdam: Limnaeus press.

Flecker, A. S. (1992). Fish tropical guilds and the structure of a tropical stream: weak direct vs strong indirect effects. Ecology, 73, 927-940.

Galvis, G., Mojica, J. I., Rodriguez, F. (1989). Estudio ecológico de una laguna de desborde del río Metica. Bogotá: Fondo FEN Colombia / Universidad Nacional de Colombia.

Gery, J. (1964). Characoids of the world. New Jersey: Neptuno.

Gorman, O.T., Karr, J. R. (1978). Habitat structure and stream fish communities. Ecology, 59, 507-515.

Guilliam, J.F., Fraser, D. L. Akins-Koo, M. (1993). Structure of a tropical stream community: a role for biotic interactions. Eco$\log y, 74,1856-1870$.

Lowe-McConell, R. (1987). Ecological studies in tropical fish communities. Cambridge: Cambridge University Press.

Mago-Leccia, F. (1994). Electric fishes of the continental waters of America. Caracas: Fudeci.

Peres, C. Gardner, T. A., Barlow, J., Zuanon, J., Michalski, F., Lees, A. C. Vieira, I. C., Moreira, F. M., Feeley, K. (2010). Biodiversity conservation in human-modified Amazonian forest landscapes. Biological Conservation, 143(10), 2314-2327.

Schultz. L.P. (1944). The catfishes of Venezuela with descriptions of thirtyeight new-forms. Washington D.C.: Smithsonian Institution.

Sterba, G. (1959). Freshwater fishes of the world. Leipzig-Jena: Ucrania-Verlag. 
Guillermo Armando Briceńo Vanegas, Julie Carolina Gallego Herrera

Cambios en la estructura de un ecosistema acuático como respuesta a disturbios antrópicos

en la selva aledaña al poblado de Atalaia do norte, Amazonas, Brasil

Thieme, B., Abell, R., Hamilton, S., Kellndorfer, J., Power, G. \& Riveros, J. C. (2007). Freshwater conservation planning in data-poor areas: An example from a remote Amazonian basin (Madre de Dios River, Peru and Bolivia). Biological Conservation, 135(4), 484-501.

Vannote, R., Minshall, G. W., Cummins, K. W., Sedell, J. R., Cushing, C. E. (1980). The River Continuum Concept. Canadian Journal of Fisheries and Aquatic Sciences, 37(1), 130-137.
Winemiller K. O. (1990). Spatial and temporal variation in tropical fish trophic networks. Ecological Monographs, 60, 331-367.

Wittman, F., Junk, W. J., Piedade, T. F. (2004). The varzea forest in Amazonia; flooding and highly dynamic geomorphology interact with natural forest succession. Ecology and Management, 196, 199-212. 


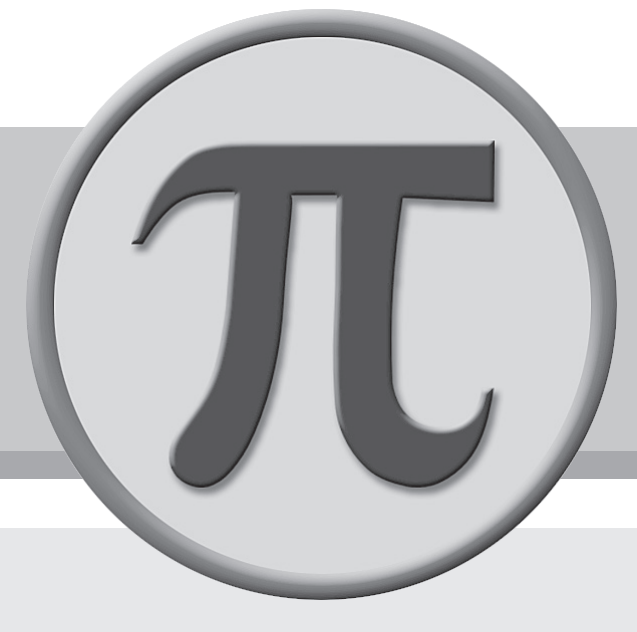


